\title{
Efeito da Interação Reprodutor x Rebanho sobre os Valores Genéticos de Reprodutores para Produção de Leite e Gordura na Raça Pardo-Suíça ${ }^{1}$
}

\author{
Rachel Santos Bueno², Robledo de Almeida Torres ${ }^{3}$, Francisco Palma Rennó2 ${ }^{2}$ José Carlos \\ Pereira $^{3}$, Cláudio Vieira de Araújo ${ }^{4}$, Paulo Sávio Lopes ${ }^{3}$, Ricardo Frederico Euclydes ${ }^{3}$
}

RESUMO - Registros de produção de leite e de gordura foram utilizados com o objetivo de verificar o efeito das interações reprodutor x rebanho e reprodutor x rebanho-ano sobre os valores genéticos de reprodutores da raça Pardo-Suíça. Os componentes de (co)variância foram estimados pelo método da máxima verossimilhança restrita, utilizando-se três modelos de características múltiplas, considerando-se como efeitos fixos grupo genético, estação de parto e classe de rebanho-ano e, como aleatórios, os efeitos de animal, de ambiente permanente, de interação reprodutor x rebanho ou reprodutor x rebanho-ano, quando considerada no modelo, e do erro. O teste da razão de verossimilhança foi aplicado, para verificação da efetividade da inclusão dos efeitos de interação nos modelos. As estimativas de componentes de (co)variâncias genética aditiva e residuais praticamente não alteraram quando os modelos foram ajustados para os efeitos de interação. Como conseqüência, os coeficientes de herdabilidade foram próximos entre si. A estimativa de herdabilidade foi de 0,40 , para ambas as características, e a correlação genética entre as características, de 0,94 , exceto quando o modelo considerou o efeito da interação reprodutor x rebanho. Neste caso, para a produção de gordura, a herdabilidade foi de 0,39 e a correlação genética entre as características de 0,95 . A proporção da variância total explicada pelas interações reprodutor x rebanho e reprodutor x rebanho-ano foram quase nulas para produção de leite, e em torno de 1\%, para produção de gordura. O logaritmo natural da função de verossimilhança aumentou quando se incluiu os efeitos de interação nos modelos. Correlações de Pearson e Spearman entre os valores genéticos obtidos pelos diferentes modelos foram superiores a 0,99, para produção de leite e de gordura, e acima de 0,897 entre as características estudadas.

Palavras-chave: bovino de leite, componentes de variância, interação genótipo-ambiente

\section{Effect of Sire x Herd Interaction on Genetic Values for Milk and Fat Yields of Brown Swiss Breed Sires}

\begin{abstract}
Records of milk and fat yields were used to study the effects of sire $\mathrm{x}$ herd and sire $\mathrm{x}$ herd-year interactions on genetic values of Brown Swiss breed sires. The (co)variance components were estimated by the restricted maximum likelihood method, by using three models with multitrait. In these models, the genetic group, season of calving and herd-year class were considered as fixed effects, while the animal effects, the permanent environment, the interaction of either sire $\mathrm{x}$ herd or sire $\mathrm{x}$ herd-year were considered as random ones, when the interaction was considered in the model, and the error as well. The likelihood ratio test was used to verify the effectiveness in including the interaction effects into models. The estimates of components of the genetic addictive and residual (co)variances did not change when the models were adjusted for the interaction effects. Therefore, the heritability coefficients approximated to each others. The heritability estimate were of 0.40 for both characteristics, and the genetic correlation among the characteristics of 0.94 , except when the model considered the effect of the interaction sire $\mathrm{x}$ herd. The heritability of fat yield was of 0.39 , and the genetic correlation among the characteristics of 0.95 . The proportion of the total variance explained by the sire $\mathrm{x}$ herd and the sire $\mathrm{x}$ herd -year interactions was low, but almost null for milk yield, and about $1 \%$ for fat yield. The natural logarithm of likelihood function increased, when the interaction effects were included in the models. Pearson and Spearman correlations among the genetic values obtained by these models were superior than 0.99 for both milk and fat yields, and above 0.897 among the studied characteristics.
\end{abstract}

Key Words: dairy cattle, genotype by environment interaction, variance components

\section{Introdução}

A avaliação genética de reprodutores leiteiros baseia-se na produção de suas filhas, envolvendo dados provenientes de rebanhos com diferentes sistemas de manejo. Quando diferenças entre progênies de um mesmo reprodutor não são as mesmas em diferentes rebanhos, ocorre a interação reprodutor x rebanho. A interação pode interferir nas variações genéticas, fenotípicas e ambientais, implicando na possibilidade 
de mudanças nos critérios de seleção dependendo do ambiente (Henderson, 1984).

Segundo Stanton et al. (1991), existem duas formas de interação genótipo $\mathrm{x}$ ambiente. Uma ocorre quando a correlação genética para a mesma característica avaliada em dois ambientes é significativamente pequena, sugerindo que a classificação dos animais baseada nos valores genéticos preditos para cada ambiente pode não ser a mesma. A outra ocorre quando a correlação genética é alta, mas a magnitude das diferenças entre os valores genéticos dos animais é diferente entre ambientes, sendo a heterogeneidade de variâncias indicada como causa desta forma de interação genótipo x ambiente.

A semelhança entre filhas de um touro em um mesmo rebanho reflete tanto a interação genótipo $\mathrm{x}$ ambiente como a covariância ambiental entre o desempenho de meio-irmãs paternas, companheiras de rebanho (Meyer, 1987; Banos \& Shook, 1990). Esta é causada por fatores diferentes do pai comum; a maior parte decorrente do tratamento diferenciado dado a filhas do mesmo reprodutor, submetidas a condições semelhantes de ambiente.

Se a interação reprodutor $\mathrm{x}$ rebanho for incluída no modelo de avaliação genética, a influência das observações provenientes de poucos rebanhos será mais limitada na avaliação dos reprodutores, podendo não afetar consideravelmente a avaliação de animais com progênies em muitos rebanhos. No entanto, ignorála aumentaria as estimativas de variância genética aditiva, o que levaria à subestimação dos valores genéticos dos reprodutores e de sua acurácia. Portanto, este efeito é mais importante para avaliação de vacas - visto que elas têm suas produções em um único rebanho (Vinson, 1987) e touros em serviço de monta natural-que de touros utilizados em inseminação artificial (Teixeira et al., 1992), pois espera-se que estes touros possuam filhas distribuídas em vários rebanhos e que a correlação de meio ambiente entre as mesmas no mesmo rebanho seja menor que aquela entre filhas em poucos rebanhos.

A existência de interação genótipo $\mathrm{x}$ ambiente tem sido testada pela estimação de componentes de variância, comparando-se modelos que incluam ou não o efeito de interação genótipo $\mathrm{x}$ ambiente (Banos \& Shook, 1990; Dimov et al., 1995). Outra maneira de se detectar a presença de interação genótipo x ambiente seria pela comparação entre as classificações dos animais, de acordo com os valores genéticos preditos, por meio dos modelos citados anteriormente (Mohammad et al., 1982; Basu \& Chattaraji, 1988). Porém, para que a mudança na classificação dos animais seja considerada efeito da interação genótipo x ambiente, é preciso que esta mudança seja significativa.

Norman (1974) recomendou a inclusão do efeito da interação reprodutor $\mathrm{x}$ rebanho no modelo estatístico, na forma de correlação ambiental, para se limitar o efeito do tratamento preferencial entre rebanhos.

Costa (1999) reportou que a estimativa da variância referente à interação reprodutor $\mathrm{x}$ rebanho tem representado em torno de 1 a $4 \%$ da variação total da produção de leite.

Este trabalho foi realizado para se estimar os componentes de variância para o efeito das interações reprodutor $\mathrm{x}$ rebanho e reprodutor $\mathrm{x}$ rebanho-ano sobre as produções de leite e de gordura do leite, em registros de lactações de vacas da raça Pardo-Suíça, e verificar a importância da inclusão destes efeitos no modelo de avaliação genética dos reprodutores.

\section{Material e Métodos}

Os dados utilizados são provenientes do Controle Leiteiro da Associação Brasileira dos Criadores de Gado Pardo-Suíça (ABCGPS), fornecidos pelo convênio mantido entre o Departamento de Zootecnia da Universidade Federal de Viçosa e a ABCGPS. O conjunto inicial de dados possuía 12.066 registros de lactações de 5.798 vacas, distribuídas em 269 rebanhos, com parições nos anos de 1980 a 2002.

Os dados foram editados para eliminação de registros de produção e ascendência incompletas, lactações encerradas por causas anormais de secagem, lactações inferiores a 150 e superiores a 450 dias, registros de produção de leite inferiores a $1.000 \mathrm{~kg}$ ou superiores a $15.000 \mathrm{~kg}$, e ainda, idade da vaca no parto menor que 18 e maior que 197 meses, considerandose somente os registros das quatro primeiras lactações, em virtude do número reduzido de observações nas lactações seguintes.

Quatro estações de parto foram estabelecidas de acordo com os meses de parto da vaca: estação 1, meses de janeiro a março; estação 2, abril a junho; estação 3, julho a setembro; e estação 4, outubro a dezembro. Com base na informação de ascendência e origem dos animais, as vacas foram classificadas em três grupos genéticos: 1) formado por vacas Pardo-Suíças puras de origem (PO); 2) vacas Pardo- 
Suíças puras por cruzamento (PC) e 3) vacas PardoSuíças puras de origem importada (POI) (animais nascidos fora do Brasil e importados). Posteriormente, os efeitos fixos de ano e rebanho foram agrupados em classes.

Para que fosse possível investigar o efeito das interações reprodutor $\mathrm{x}$ rebanho e reprodutor $\mathrm{x}$ rebanho-ano, exigiu-se que cada reprodutor possuísse, no mínimo, quatro filhas em dois diferentes rebanhos e que cada classe de rebanho-ano tivesse, no mínimo, quatro observações. Após eliminações, o arquivo de dados para análise constituiu de 8.566 lactações, provenientes de 4.318 vacas, filhas de 152 reprodutores, as quais pariram nos anos de 1980 a 2000, em 138 rebanhos.

As lactações foram ajustadas previamente para número de ordenhas (duas ordenhas - 2x), duração da lactação (305 dias) e para a produção na idade adulta da vaca (72 e 69 meses de idade, para produção de leite e gordura, respectivamente), de acordo com os ajustes obtidos por Rennó (2001).

A estimação de componentes de (co)variâncias, parâmetros genéticos e a predição dos valores genéticos foram feitas utilizando o programa MTDFREML (Multiple Trait Derivative Free Restricted Maximum Likelihood), descrito por Boldman et al. (1995), com modelo animal. O critério de convergência da variância dos valores do 'simplex' adotado foi de, no mínimo, $10^{-9}$.

As análises foram conduzidas utilizando-se três modelos de características múltiplas, os quais consideraram como efeitos fixos grupo genético, estação de parto e rebanho-ano de parto e, como aleatórios, os efeitos de animal, de ambiente permanente, das interações reprodutor $\mathrm{x}$ rebanho ou reprodutor $\mathrm{x}$ rebanho-ano, quando consideradas no modelo, e do erro e diferiam em relação à: ausência do termo da interação, inclusão da interação reprodutor x rebanho e modelo com a interação reprodutor $\mathrm{x}$ rebanho-ano.

Em notação matricial, o modelo que considera o efeito de interação reprodutor $\mathrm{x}$ rebanho-ano foi definido como:

$$
Y_{\sim}=X_{i} \beta_{\sim}+Z_{a i} a_{i}+Z_{p i} p_{\sim}+Z_{(s x h x y) i} t_{\sim}+e_{i}
$$

em que: $\underset{\sim}{Y_{i}}=$ vetor $\mathrm{n}_{\mathrm{i}} \times 1$, de $\mathrm{n}_{\mathrm{i}}$ observações da característica $\mathrm{i}, \mathrm{i}=$ produção de leite (1) e produção de

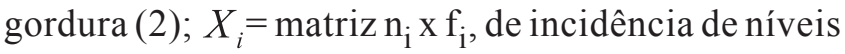

dos efeitos fixos na característica $i ; \beta_{i}=$ vetor $f_{i} \times 1$, de efeitos fixos referentes à característica $\mathrm{i} ; Z_{a i}=$ matriz diagonal $n_{i} \times N_{i}$, de incidência dos valores genéticos, contendo " 1 " na diagonal para a característica i; $\underset{\sim}{a_{i}}=$ vetor $\mathrm{N}_{\mathrm{i}} \times 1$, de valores genéticos dos animais na característica $\mathrm{i} ; Z_{p i}=$ matriz diagonal $\mathrm{n}_{\mathrm{i}} \mathrm{x}$ $\mathrm{N}_{\mathrm{i}}$, de incidência dos efeitos de ambiente permanente dos animais, na característica $\mathrm{i} ; \underset{\sim}{p}{\underset{\sim}{ }}_{=}$vetor $\mathrm{N}_{\mathrm{i}} \times 1$, de valores referentes ao efeito de ambiente permanente dos animais na característica $\mathrm{i} ; Z_{(s x h x) i}=$ matriz de dimensão $n_{\mathrm{i}} \mathrm{x}_{\mathrm{i}}$, de incidência dos efeitos da interação reprodutor $\mathrm{x}$ rebanho-ano, composta de zeros e uns; ${ }_{\sim}{ }_{i}$ $=$ vetor $\mathrm{v}_{\mathrm{i}} \times 1$, de valores referentes ao efeito da interação reprodutor $x$ rebanho-ano na característica

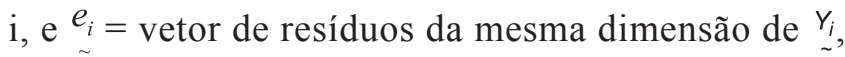
sendo $\mathrm{N}=$ número de indivíduos, $\mathrm{n}=$ número total de observações, $\mathrm{f}=$ número de classes de efeitos fixos, $\mathrm{e} \mathrm{v}=$ número de níveis da interação reprodutor $\mathrm{x}$ rebanho-ano.

Considerando-se conta uma distribuição conjunta das características, têm-se:

$$
\begin{gathered}
\underset{\sim}{Y}=\left[\begin{array}{c}
Y_{1} \\
\sim \\
Y_{2} \\
\sim
\end{array}\right], \quad X=\left[\begin{array}{cc}
X_{1} & \phi \\
\tilde{\phi} & X_{2}
\end{array}\right], \quad \beta=\left[\begin{array}{c}
\beta_{1} \\
\sim \\
\beta_{2} \\
\sim
\end{array}\right] \\
Z_{a}=\left[\begin{array}{cc}
Z_{a 1} & \phi \\
\phi & Z_{a 2}
\end{array}\right], \quad Z_{p}=\left[\begin{array}{cc}
Z_{p 1} & \phi \\
\phi & Z_{p 2}
\end{array}\right], \quad Z_{s x k x y}=\left[\begin{array}{cc}
Z_{(x k k x y) l} & \phi \\
\phi & Z_{(s k h x y) 2}
\end{array}\right] \\
\underset{\sim}{a}=\left[\begin{array}{c}
a_{1} \\
\sim \\
a_{2} \\
\sim
\end{array}\right], \quad \underset{\sim}{p}=\left[\begin{array}{c}
p_{1} \\
\sim \\
p_{2} \\
\sim
\end{array}\right], \quad t=\left[\begin{array}{c}
t_{1} \\
\sim \\
t_{2} \\
\sim
\end{array}\right], \quad \underset{\sim}{e}=\left[\begin{array}{c}
e_{1} \\
\sim \\
e_{2} \\
\sim
\end{array}\right]
\end{gathered}
$$

em que: $Y_{1}=$ produção de leite e $Y_{2}=$ produção de gordura.

Admitindo-se que $\underset{\sim}{Y}, \underset{\sim}{a}, p, \underset{\sim}{t}$ e $\underset{\sim}{e}$ tenham distribuição normal multivariada,

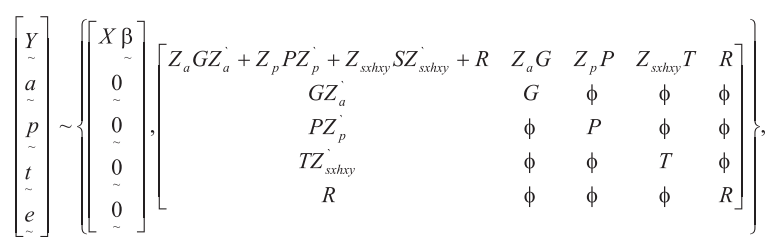

tem-se que: $G=A \otimes G_{0}, \quad P=I_{N} \otimes P_{0}, \quad T=I_{v} \otimes T_{0}$ e $R=I_{n} \otimes R_{0}$, sendo: $A=$ matriz de numeradores do 
coeficiente de parentesco entre os indivíduos, de ordem igual ao número total de indivíduos $(\mathrm{N}) ; G_{0}=$ matriz $2 \times 2$, de variância e covariância genética aditiva entre as características avaliadas; e $\otimes=$ operador produto direto (Searle, 1966).

$$
G_{0}=\left[\begin{array}{ll}
\sigma_{a 11}^{2} & \sigma_{a 12} \\
\sigma_{a 21} & \sigma_{a 22}^{2}
\end{array}\right],
$$

em que: $\sigma_{\text {aii }}^{2}=$ variância genética aditiva da característica i, e $\sigma_{a i j}$, covariância genética aditiva entre as características; $\mathrm{I}_{\mathrm{N}}$, matriz identidade de $\operatorname{ordem} \mathrm{N}$, e

$$
P_{0}=\left[\begin{array}{cc}
\sigma_{p 11}^{2} & 0 \\
0 & \sigma_{p 22}^{2}
\end{array}\right] ;
$$

em que: $\sigma_{\text {aii }}^{2}=$ variância referente ao efeito permanente de meio ambiente da característica i; $I_{\mathrm{v}}=$ uma matriz identidade de ordem $\mathrm{v}$, e

$$
T_{0}=\left[\begin{array}{cc}
\sigma_{(s x h x y) 11}^{2} & 0 \\
0 & \sigma_{(s x h x y) 22}^{2}
\end{array}\right] ;
$$

em que: $\sigma_{(s x h x y) i i}^{2}=$ variância referente à interação reprodutor $\mathrm{x}$ rebanho-ano, para a característica $\mathrm{i}$; $\mathrm{I}_{\mathrm{n}}=$ matriz identidade de ordem $n$, e

$$
R_{0}=\left[\begin{array}{ll}
\sigma_{e 11}^{2} & \sigma_{e 12} \\
\sigma_{e 21} & \sigma_{e 22}^{2}
\end{array}\right],
$$

em que: $\sigma_{e i i}^{2}=$ variância residual da característica i, e $\sigma_{e i j}=$ covariância residual entre as características.

As equações do modelo misto [3] são descritas como:

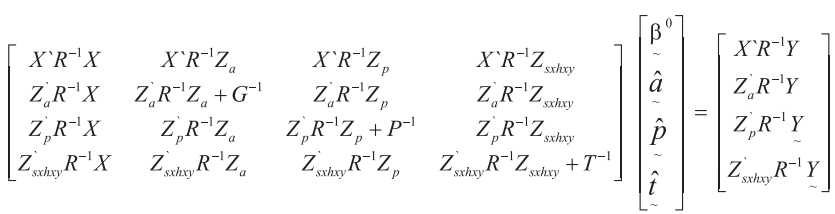

Nas análises que consideram o efeito da interação reprodutor $\mathrm{x}$ rebanho, os modelos são semelhantes aos descritos anteriormente, com a inclusão da matriz $Z_{(s x h) i}$ e do vetor ${ }_{\sim} s_{i}$, em substituição ao componente da interação reprodutor x rebanho-ano, em que: $Z_{(s x h) i}$ é uma matriz de incidência de dimensão $\mathrm{n}_{\mathrm{i}} \mathrm{x} \mathrm{h}_{\mathrm{i}}$, referente aos efeitos da interação reprodutor $\mathrm{x}$ rebanho, na característica i; e ${ }_{i}$ é um vetor de dimensão $h_{\mathrm{i}}$ x 1 , ou seja, igual ao número de níveis de interação reprodutor $\mathrm{x}$ rebanho na característica i. As análises sem inclusão do efeito de interação diferem destas apenas pela ausência dos componentes desses efeitos nos modelos.
A importância da inclusão do efeito das interações reprodutor $\mathrm{x}$ rebanho ou reprodutor $\mathrm{x}$ rebanho-ano no modelo de avaliação genética dos animais foi verificada pelo teste da razão de verossimilhança de modelos seqüencialmente reduzidos (Rao, 1973). A estimativa da estatística do teste da razão de verossimilhança (LR) foi comparada com o valor obtido pela distribuição de qui-quadrado $\left(X_{t a b}^{2}\right)$, com 1 grau de liberdade e nível de significância fixo de $1 \%$.

Os valores genéticos preditos dos reprodutores para as produções de leite e gordura foram organizados em arquivos, com a finalidade de se verificar possíveis alterações na magnitude das predições dos valores genéticos e no ordenamento dos reprodutores baseado nestas predições, quando a interação reprodutor $\mathrm{x}$ rebanho ou reprodutor $\mathrm{x}$ rebanho-ano foi incluída no modelo, por meio das correlações de Pearson e Spearman, empregando-se o pacote estatístico Statistical Analysis System (SAS, 1999).

\section{Resultados e Discussão}

As médias observadas e os desvios-padrão para produção de leite e de gordura do leite foram de 5.959,86 \pm $1.733,21$ e 223,23 $\pm 64,72$, respectivamente.

Os componentes de (co)variâncias estimados pelos modelos com e sem interação reprodutor x rebanho ou reprodutor $\mathrm{x}$ rebanho-ano são apresentados na Tabela 1. As estimativas de componentes de variância genética aditiva e residual para produção de leite apresentaram variações inferiores a $1 \%$ com a inclusão do efeito de interação no modelo, aumentando em $0,99 \%$ e $0,10 \%$ quando o efeito da interação reprodutor $\mathrm{x}$ rebanho foi considerado, e reduzindo em $0,43 \% \mathrm{e}$ $0,96 \%$, com o efeito da interação reprodutor x rebanho-ano, respectivamente. Para a característica produção de gordura, houve redução de $2,74 \%$ e aumento de $0,66 \%$, com a inclusão do efeito das interações reprodutor x rebanho e reprodutor x rebanho-ano, respectivamente, no componente de variância genética aditiva, e reduções inferiores a $1 \%$ nas estimativas de componentes de variâncias residuais. Não havendo praticamente alteração dos componentes de covariância genética aditiva e residual quando o modelo foi ajustado para os efeitos de interação.

O componente de variância de ambiente permanente teve redução de $16,07 \%$, para a característica produção de leite, com a inclusão no modelo do efeito 
Tabela 1 - Componentes de (co)variância genética aditiva, ambiente permanente, interação e residual para produção de leite e de gordura, obtidos pelos modelos sem e com interação

Table 1 - Additive genetic, permanent environment, interaction and residual (co)variance components for milk and fat yield in a model without and with interaction

\begin{tabular}{|c|c|c|c|c|c|c|}
\hline & & & \multicolumn{4}{|c|}{$\begin{array}{l}\text { Modelo com interação } \\
\text { Model with interaction }\end{array}$} \\
\hline & \multicolumn{2}{|c|}{$\begin{array}{l}\text { Modelo sem interação } \\
\text { Model without interaction }\end{array}$} & \multicolumn{2}{|c|}{$\begin{array}{l}\text { Reprodutor x rebanho } \\
\text { Sire by herd }\end{array}$} & \multicolumn{2}{|c|}{$\begin{array}{l}\text { Reprodutor x rebanho-ano } \\
\text { Sire by herd-year }\end{array}$} \\
\hline & $\begin{array}{l}\text { Leite } \\
\text { Milk }\end{array}$ & $\begin{array}{l}\text { Gordura } \\
\text { Fat }\end{array}$ & $\begin{array}{l}\text { Leite } \\
\text { Milk }\end{array}$ & $\begin{array}{l}\text { Gordura } \\
\text { Fat }\end{array}$ & $\begin{array}{l}\text { Leite } \\
\text { Milk }\end{array}$ & $\begin{array}{c}\text { Gordura } \\
\text { Fat }\end{array}$ \\
\hline $\begin{array}{l}\text { Genética aditiva }\left(\mathrm{kg}^{2}\right) \\
\text { Additive genetic effect }\left(\mathrm{kg}^{2}\right) \\
\text { Leite }(\text { milk }) \\
\text { Gordura }(\text { fat }) \\
\end{array}$ & $585.794,19003$ & $\begin{array}{c}21.442,47512 \\
882,15910\end{array}$ & $591.606,84465$ & $\begin{array}{c}21.295,34486 \\
857,94070\end{array}$ & $583.242,94189$ & $\begin{array}{c}21.457,17971 \\
888,06763 \\
\end{array}$ \\
\hline $\begin{array}{l}\text { Efeito permanente de meio } \\
\text { Permanent environment effec } \\
\text { Leite (milk) } \\
\text { Gordura (fat) }\end{array}$ & $\begin{array}{l}\left.\mathrm{kg}^{2}\right) \\
\left(\mathrm{kg}^{2}\right) \\
33.101,57377\end{array}$ & $\begin{array}{l}0,00000 \\
7,07542 \\
\end{array}$ & $27.781,66645$ & $\begin{array}{l}0,00000 \\
0,00015\end{array}$ & $36.311,89839$ & $\begin{array}{l}0,00000 \\
0,00035\end{array}$ \\
\hline $\begin{array}{l}\text { Efeito de interação }\left(\mathrm{kg}^{2}\right) \\
\text { Interaction effect }\left(\mathrm{kg}^{2}\right) \\
\text { Leite (milk) } \\
\text { Gordura (fat) }\end{array}$ & & & 0,09481 & $\begin{array}{c}0,00000 \\
25,79979\end{array}$ & $7.601,37983$ & $\begin{array}{c}0,00000 \\
16,64011\end{array}$ \\
\hline $\begin{array}{l}\text { Efeito residual }\left(\mathrm{kg}^{2}\right) \\
\text { Residual effect }\left(\mathrm{kg}^{2}\right) \\
\text { Leite (milk) } \\
\text { Gordura (fat) }\end{array}$ & $857.098,69944$ & $\begin{array}{c}29.888,25413 \\
1.311,89126\end{array}$ & $857.997,28997$ & $\begin{array}{c}29.869,08417 \\
1.307,95823\end{array}$ & $848.837,34154$ & $\begin{array}{c}29.861,460 \\
1.299,16738\end{array}$ \\
\hline
\end{tabular}

da interação reprodutor x rebanho, e aumentou em $9,7 \%$ no modelo que considerava a interação reprodutor x rebanho-ano. Para produção de gordura, as estimativas foram próximas de zero, quando considerados nos modelos os efeitos de interação.

Reduções nas estimativas de componentes de variâncias, quando considerado nas análises o efeito da interação reprodutor $\mathrm{x}$ rebanho, foram observadas por Tong et al. (1977), Meyer (1987) e Banos \& Shook (1990).

Geraldini (2002), estudando o efeito da interação reprodutor $\mathrm{x}$ rebanho sobre estimativas de componentes de variância e sobre a avaliação genética de reprodutores na raça Pardo-Suíça, ao incluir o termo de interação reprodutor x rebanho no modelo estatístico, verificou reduções nas estimativas de componentes de variância referentes ao efeito genético aditivo de, aproximadamente, 1 e $4 \%$ para produções de leite e gordura, respectivamente, e de $1 \%$ na estimativa do componente de covariância entre as características. A variância do efeito permanente de meio apresentou redução de $24,36 \%$, para a característica produção de leite, e foi quase nula, para ambos os modelos, com e sem interação para produção de gordura. Os componentes de (co)variâncias residuais praticamente não alteraram, com reduções inferiores a $0,1 \%$ para ambas as características.

As estimativas dos componentes de variância referentes aos efeitos de interação foram relativamente baixas. Algum viés poderia ser encontrado nestas estimativas, pois parte do parentesco entre os reprodutores não foi considerada, subestimando os componentes de variância. No entanto, segundo Van Tassell \& Berger (1994), essa subestimação deve ser relativamente pequena, uma vez que há pouca perda de informação ao desconsiderar o parentesco entre os reprodutores quando o nível do efeito da interação é relativamente baixo.

Como conseqüência da pequena diferença nas estimativas dos componentes de (co)variância genética aditiva e residual entre os modelos, as estimativas de herdabilidade, correlações genéticas e proporções da variância total em razão das variâncias dos efeitos de ambiente permanente, interações reprodutor $\mathrm{x}$ rebanho e reprodutor $\mathrm{x}$ rebanho-ano, quando consideradas no modelo, e residuais (Tabela 2) foram próximas entre si. Para ambas as características, produção de leite e de gordura, as estimativas de herdabilidade não variaram entre os modelos. 
Estimativas de herdabilidades deste estudo foram similares às obtidas por Geraldini (2002) e maiores que as encontradas por Meyer (1987), para produção de leite e de gordura, de 0,28 e 0,33, respectivamente, utilizando registros de novilhas de diferentes grupos genéticos da raça Holandesa. Banos \& Shook (1990) relataram estimativas de herdabilidade de 0,20 , em média, para produção de leite em vacas holandesas.

As proporções da variância total, referentes às variâncias dos efeitos de ambiente permanente e residual, praticamente não diferiram entre os modelos sem e com interação. Quanto ao componente de variância do efeito de interações reprodutor $\mathrm{x}$ rebanho e reprodutor x rebanho-ano, a proporção da variância total foi de pequena magnitude, sendo próxima de zero para produção de leite e em torno de 1\% para produção de gordura. Esses resultados foram inferiores aos encontrados por Lee (1976), Tong et al. (1977), Meyer (1987) e Banos \& Shook (1990), em que os componentes de variância de interação representaram de 2 a 4\% da variância total.

Segundo Kelleher et al. (1967), sempre que o componente de variância for diferente de zero, os efeitos de interação causarão erros, mas a magnitude deste erro se torna importante na avaliação de reprodutores quando o componente de variância da interação for responsável por mais de $10 \%$ da variância total. Assim, outros fatores (genéticos, de meio e de erro) influenciam mais significativamente a acurácia de seleção que os efeitos de interação. O aumento no número de filhas de touros de inseminação artificial também tenderá a reduzir a influência de interação junto aos erros causados por outros fatores.

A inclusão da interação reprodutor $\mathrm{x}$ rebanho ou de reprodutor $\mathrm{x}$ rebanho-ano nos modelos não provocou consideráveis alterações nas estimativas de componentes de variância. Contudo, esses efeitos, quando considerados nas análises, provocaram aumentos significativos $(\mathrm{P}<0,01)$ no logaritmo natural da função de verossimilhança, conforme indicado pelo teste da razão de verossimilhança, para modelos seqüencialmente reduzidos (Tabela 3), indicando superioridade do modelo, quando se incluiu o efeito de interação. Meyer (1987) também verificou que os valores do logaritmo da função de verossimilhança em modelos que apresentavam interações reprodutor $\mathrm{x}$ rebanho e reprodutor x rebanho-ano-estação de parto foram significativamente maiores que para o modelo que desconsiderou essas interações.

As médias dos valores genéticos preditos para os reprodutores, os desvios-padrão, os valores mínimos e máximos e as amplitudes obtidas para as caracterís-

Tabela 2 - Estimativas de herdabilidades $\left(h^{2}\right)$, correlações genéticas $\left(r_{g}\right)$ e proporções da variância total, em razão da variância do efeito permanente de meio $\left(C^{1}\right)$, dos efeitos das interações reprodutor $x$ rebanho $\left(C^{2}\right)$ e reprodutor $\mathrm{x}$ rebanho-ano $\left(\mathrm{C}^{3}\right)$ e do efeito residual (e), para produção de leite e de gordura do leite

Table 2 - Estimates of heritability $\left(h^{2}\right)$, genetic correlation $\left(r_{g}\right)$ and proportions of the phenotypic variance due to the permanent environment $\left(C^{1}\right)$, sire by herd interaction $\left(C^{2}\right)$, sire by herd-year interaction $\left(C^{3}\right)$, and residual variance $(e)$ estimates for milk and fat yield

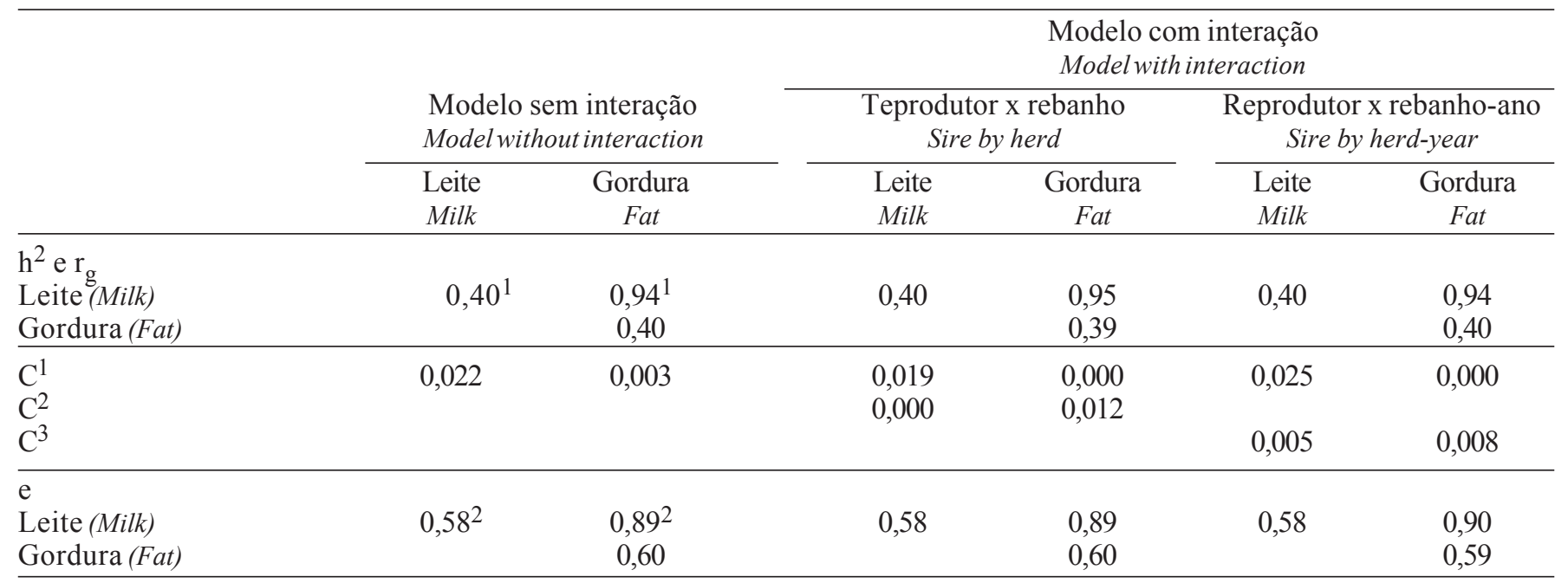

1 Herdabilidades na diagonal, correlações genéticas acima da diagonal.

2 Variâncias na diagonal, covariâncias acima da diagonal.

1 Heritability on the diagonal, genetic correlation above the diagonal.

2 Variances on the diagonal, covariances above the diagonal. 
Tabela 3 - Valores do logaritmo natural das funções de verossimilhança, para modelos sem e com interações reprodutor $x$ rebanho e reprodutor $x$ rebanho-ano, e teste da razão de verosimilhança (LR)

Table 3 - Values of the natural logarithm of the likelihood functions for models without and with sire $x$ herd and sire $x$ herd-year interactions, and likelihood ratio test (LR)

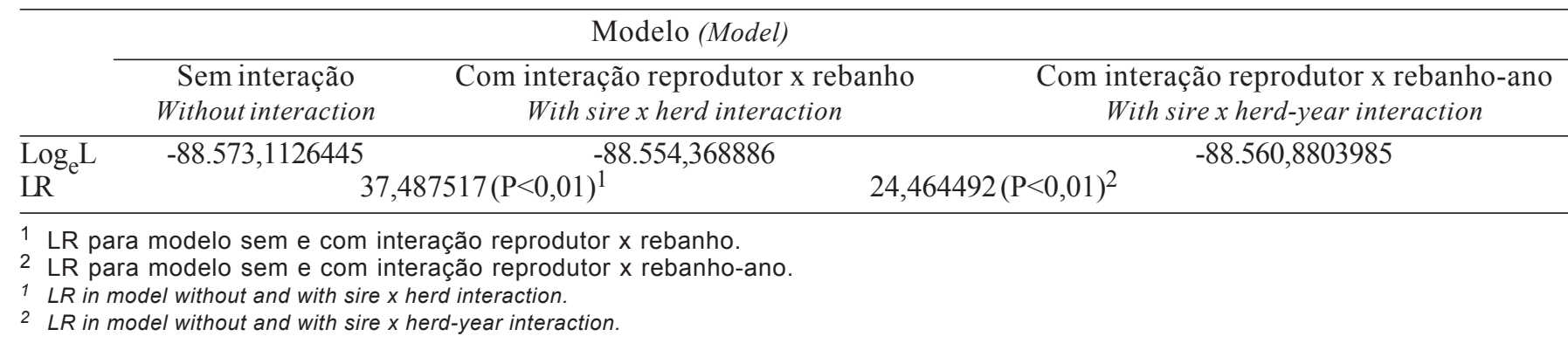

ticas produção de leite e de gordura do leite, obtidas pelos modelos sem e com interações reprodutor $\mathrm{x}$ rebanho e reprodutor $\mathrm{x}$ rebanho-ano encontram-se na Tabela 4. A amplitude dos valores genéticos preditos dos reprodutores praticamente não se alterou quando os efeitos de interação foram incluídos no modelo, para as duas características estudadas.

Os altos valores, acima de 0,99 , para as correlações de Pearson e Spearman (Tabela 5) entre os valores genéticos preditos dos reprodutores, para produção de leite e de gordura, indicam que o ordenamento dos reprodutores não foi alterado com a inclusão dos efeitos de interação nos modelos em análise. Correlações de Pearson e Spearman entre as características produção de leite e de gordura, com e sem interação, foram todas acima de 0,89 , indicando que, quando os valores genéticos preditos se basearem na produção de leite, os reprodutores seriam classificados de forma similar para ambas as características.

Carabaño et al. (1990), ao estudarem o desempenho de vacas da raça Holandesa, filhas de um mesmo reprodutor, em diferentes regiões dos Estados Unidos, estimaram coeficientes de correlações genéticas superiores a 0,90. Mohammad et al. (1982) obtiveram altas correlações de Pearson e Spearman (acima de 0,99) entre os valores genéticos preditos de modelos incluindo e ignorando o efeito da interação reprodutor $\mathrm{x}$ rebanho ou reprodutor $x$ peso ideal da novilha e concluíram que ignorar o efeito da interação não causaria maiores alterações na classificação dos reprodutores. Rorato et al. (1994), ao estimarem correlações genéticas entre os valores genéticos para um mesmo touro em diferentes regiões do estado do Paraná, concluíram que as filhas de um mesmo touro apresentavam comportamento diferenciado nos distintos ambientes, caracterizando a interação genótipo $\mathrm{x}$ ambiente.
Tabela 4 - Médias, desvios-padrão, valores mínimos, máximos e amplitudes dos valores genéticos preditos dos reprodutores para produção de leite e gordura, utilizando-se os modelos sem e com interações reprodutor $x$ rebanho e reprodutor $\mathrm{x}$ rebanho-ano

Table 4 - Means, standard deviation, minimum, maximum and range of sires breeding values predicted for milk and fat yield, using the models without and with sire by herd interaction and sire by herd-year interaction

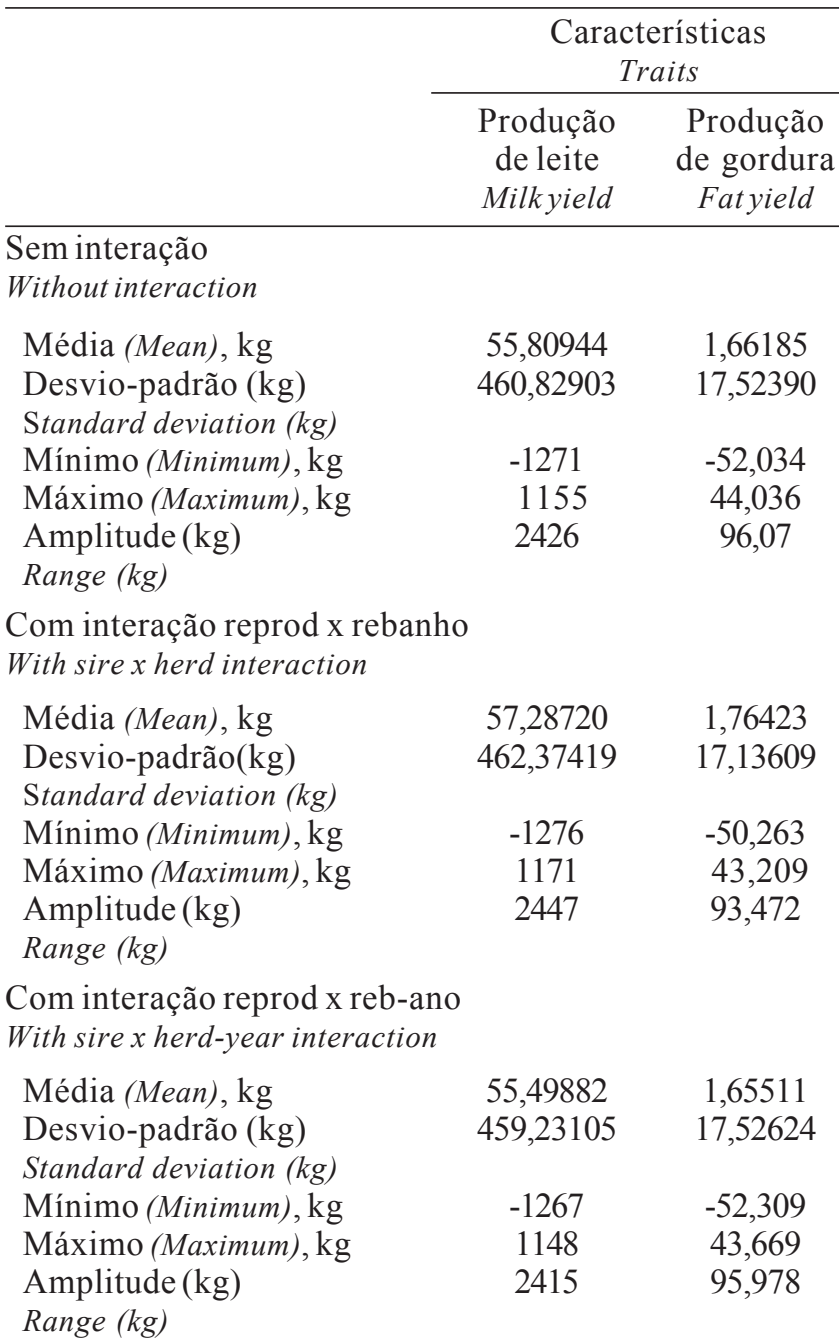


Tabela 5 - Correlações de Pearson e Spearman entre os valores genéticos preditos dos reprodutores para produção de leite e de gordura, utilizando-se os modelos sem e com interações reprodutor $x$ rebanho e reprodutor $x$ rebanho-ano

Table 5 - Pearson and Spearman correlations among sire breeding values predicted to milk and fat yield in a model without interaction and with sire $x$ herd interaction and sire $x$ herd-year interaction

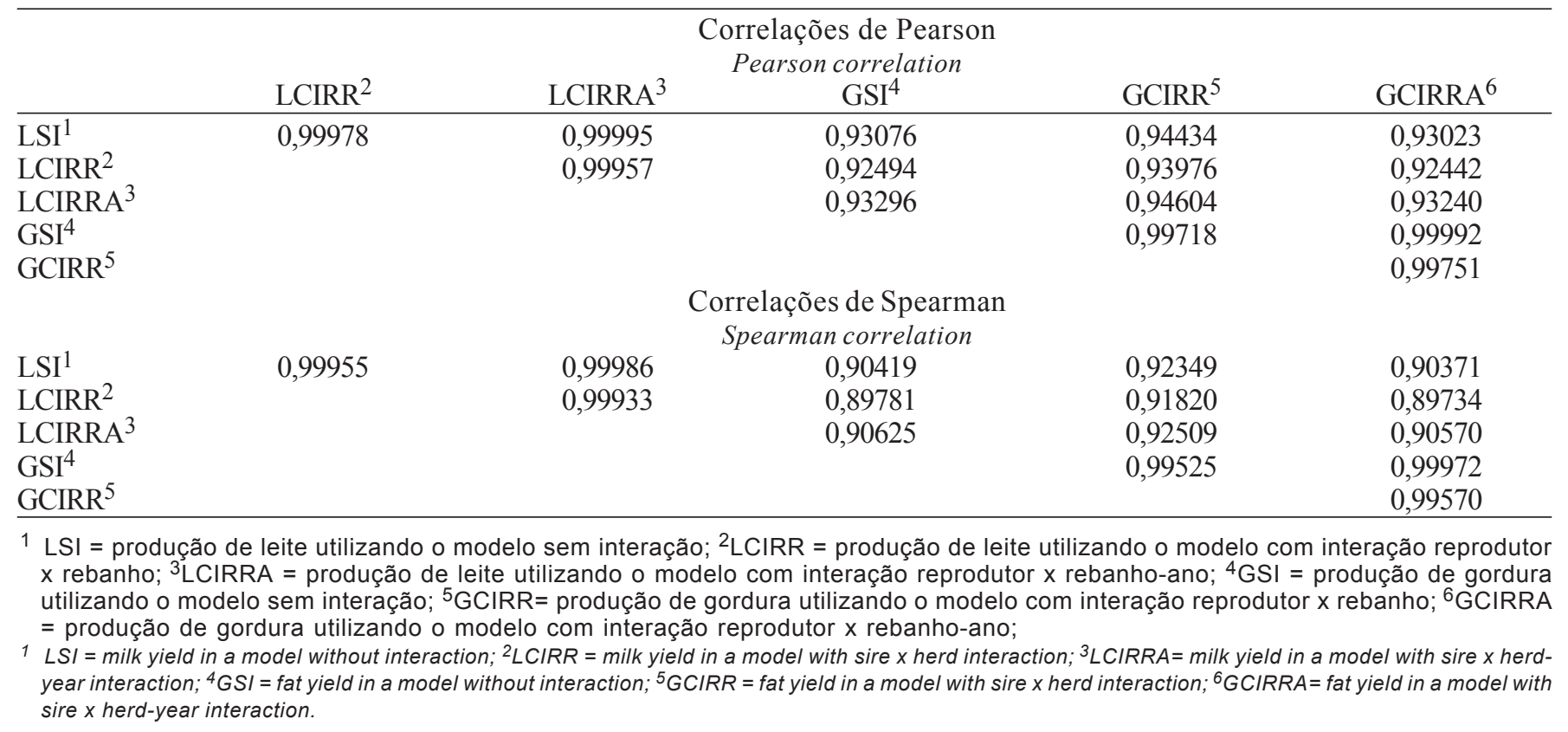

\section{Conclusões}

A inclusão dos efeitos de interação reprodutor $\mathrm{x}$ rebanho ou reprodutor $\mathrm{x}$ rebanho-ano nos modelos de avaliação genética pouco alterou as estimativas de componentes de (co)variâncias genética aditiva e residuais e, conseqüentemente, as herdabilidades. Os coeficientes de correlações de Pearson e Spearman obtidos foram altos, indicando que os reprodutores foram classificados de forma semelhante pelos seus valores genéticos preditos nos três modelos utilizados. Assim, os efeitos da interações reprodutor $\mathrm{x}$ rebanho e reprodutor $\mathrm{x}$ rebanho-ano podem ser desconsiderados na avaliação genética da raça Pardo-Suíça, para as características produção de leite e produção de gordura do leite.

\section{Agradecimento}

À Associação Brasileira de Criadores de Gado Pardo-Suíço, pelo fornecimento dos dados utilizados nesta pesquisa.

\section{Literatura Citada}

BANOS, G.; SHOOK, G.E. Genotype by environment interaction and genetic correlations among parities for somatic cell count and milk yield. Journal of Dairy Science, v.73, n.9, p.2563-2573, 1990.

BASU, S.B.; CHATTARAJI, J. Genotype-environment interaction in the evaluation of sires of crossbred cows. Indian Journal of Animal Science, v.58, n.7, p.808-811, 1988.

BOLDMAN, K.G.; KRIESE, L.A.; Van VLECK, L.D. et al. A manual for use of MTDFREML: a set of programs to obtain estimates of variances and covariances (DRAFT). Lincoln: Department of Agriculture/Agriculture Research Service, 1995. $125 \mathrm{p}$.

CARABAÑO, M.J.; WADE, K.M.; Van VLECK, L.D. Genotype by environment interactions for milk and fat production across regions of United States. Journal of Dairy Science, v.73, n.1, p.173-180, 1990.

COSTA, C.N. Interação genótipo x ambiente em gado de leite. In: SIMPÓSIO INTERNACIONAL DE GENÉTICA E MELHORAMENTO ANIMAL, 1., 1999, Viçosa, MG. Anais... Viçosa, MG: Universidade Federal de Viçosa, 1999. p.161-191.

DIMOV, G.; ALBUQUERQUE, L.G.; KEOWN, J.F. et al. Variance of interaction effects of sire and herd for yield traits of Holsteins in California, New York, and Pennsylvania with an animal model. Journal of Dairy Science, v.78, n.4, p.939-946, 1995. 
GERALDINI, M.L.F. Efeito da interação reprodutor $\mathbf{x}$ rebanho sobre a produção e gordura do leite da raça PardoSuíça. Viçosa, MG: Universidade Federal de Viçosa, 2002. 68p. Dissertação (Mestrado em Zootecnia) - Universidade Federal de Viçosa, 2002.

HENDERSON, C.R. Aplication of linear models in animal breeding. University of Guelph, 1984. 462p.

KELLEHER, D.J.; FREEMAN, A.E.; LUSH, J.L. Importance of bull $\mathrm{x}$ herd-year-season interaction in milk production. Journal of Dairy Science, v.50, n.10, p.1703-1707, 1967.

LEE, A.J. Relation between milk yield and age at first calving in first lactation. Journal of Dairy Science, v.59, n. 10, p.1794$1801,1976$.

MEYER, K. Estimates of variance due to sire $x$ herd interactions and environmental covariances between paternal half-sibs for first lactation dairy production. Livestock Production Animal Science, v.17, n.1, p.95-115, 1987.

MOHAMMAD, W.A.; LEE, A.J.; GROSSMAN, M. Genotypeenvironment interaction in sire evaluation. Journal of Dairy Science, v.65, n.5, p.857-860, 1982.

NORMAN, H.D. Factors that should be considered in a national sire summary model. Journal of Dairy Science, v.57, n.7, p.955-962, 1974.

RAO, C.R. Linear statistical inference and its aplications. 2.ed. New York: John Wiley \& Sons, 1973. 552p.

RENNÓ, F.P. Aspectos produtivos da raça Pardo-Suíça no Brasil. Viçosa, MG: Universidade Federal de Viçosa, 2001. 100 p. Dissertação (Mestrado em Zootecnia) - Universidade Federal de Viçosa, 2001.

RORATO, P.R.N.; LÔBO, R.B.; MARTINS FILHO, R. et al Efeito da interação genótipo-ambiente sobre a produção de leite da raça Holandesa, no estado do Paraná. Revista da Sociedade Brasileira de Zootecnia, v.23, n.5, p.859-869, 1994.
SEARLE, S.R. Matrix algebra for biological science. New York: John Wiley and Sons, 1966. 296p.

STANTON, T.L.; BLAKE, R.W.; QUAAS, R.L. et al. Genotype by enviroment interaction for Holstein milk yield in Colombia, México and Porto Rico. Journal of Dairy Science, v.74, n.5, p.1700-1714, 1991.

STATISTICAL ANALYSES SYSTEM - SAS. User's guide. version 8.0. Cary: 1999.

TEIXEIRA, N.M.; FREITAS, A.F.; VALENTE, J. Correlação de meio-ambiente entre meio-irmãs paternas para a produção de leite na raça Holandesa. Revista da Sociedade Brasileira de Zootecnia, v.21, n.3, p.501-509, 1992.

TONG, A.K.W.; KENNEDY, B.W.; MOXLEY, J.E. Sire by herd interactions for milk yield and composition traits. Canadian Journal of Animal Science, v.57, n.3, p.383-388, 1977.

VAN TASSELL, C.P.; BERGER, P.J. Consideration of sire relationships for estimation of variance components with interaction of herd and sire. Journal of Dairy Science, v.77, n.1, p.313-324, 1994.

VINSON, W.E. Potential bias in genetic evaluations from differences in variation within herds. Journal of Dairy Science, v.70, n.9, p.2450-2455, 1987. 